\title{
From a rod to a disk
}

\author{
Paul Seiler, Klaus Wallmeroth and Kurt Mann
}

\section{The Nd:YAG was one of the first ever industrial lasers, and even today it still has many advantages over other laser technologies. Competition from newer laser technologies, however, has made its evolution critical to its survival.}

The year 1964 is recorded as being the birth of the Nd:YAG laser. Made from an yttrium aluminium garnet (YAG) crystal doped with neodymium, Nd:YAG has optical and mechanical properties similar to ruby, which was used in the first demonstration of lasing in 1960. However, Nd:YAG has a much lower excitation threshold than ruby and can therefore be operated at room temperature in continuous-wave (CW) mode. This represented a huge breakthrough in the development of laser technology. The optical resonator and pump arrangement (including the lamp, reflector and electrical supply) were well known from earlier ruby laser technology, and this allowed the ruby rod to be simply replaced by the Nd:YAG rod.

Today, YAG lasers - doped not only with neodymium but also with other rare earth elements - are used as tools in materials processing, ranging from precision engineering at the microscale to the welding of ship panels. But these lasers are not just significant for materials processing, even though this is their primary focus; they are also used in the medical industry, in measurement technology and in the military.

There are three properties that make this type of laser particularly useful: the high amplification of laser light; the option to store energy in the active medium; and the wavelength of operation $(1,064 \mathrm{~nm})$.

Single-rod YAG lasers allow pulses of several kilowatts in power and durations of several milliseconds to be produced. Although the first lasers had average outputs of less than $10 \mathrm{~W}$, the high level of amplification offered by YAG made it possible to produce small spotwelds. Today, pulsed lasers for spot welding have average outputs of 20-500 W for a multitude of applications. Another interesting feature of early pulsed lasers was the introduction of Q-switching - a shuttering scheme for the creation of large laser pulses - for distance measurements in the military. Q-switching is able to provide pulses with powers in the megawatt range (with corresponding nanosecond pulse durations), and was later used to write information on metal/plastic media. More recently, ultrashortpulse YAG lasers in the picosecond range have

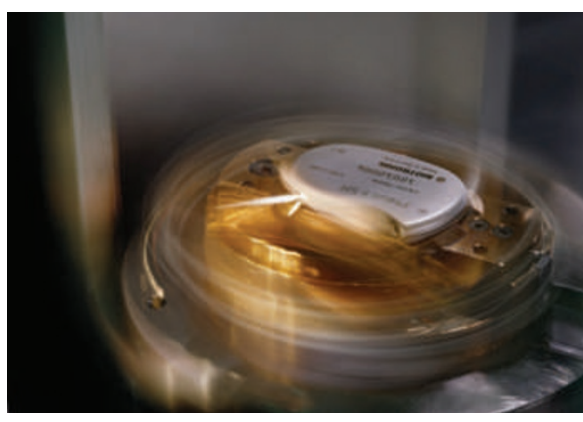

With its ability to weld and cut many different materials, the Nd:YAG laser has become the workhorse of many industries.

been introduced for applications involving high-precision processing.

Advantageously, the use of fibre-optic guiding made it possible to separate the laser from the processing location. This means that one laser can be used in multiple locations, either simultaneously, by splitting the laser into multiple fibres, or by time-sharing, with the help of an optical switch. Connecting the laser to a robotic head via the fibre optics enables 3D processing, with the laser moving around the object.

The kilowatt CW YAG laser was adopted for use in large-scale industrial applications in the early 1990s, but this would not have been possible without the flexibility of fibreoptic delivery. Today, these lamp-pumped multi-kilowatt lasers are still running very successfully in the automotive industry. Nevertheless, new solid-state laser concepts based on diode pumping - the fibre laser and the disk laser - have been developed since the mid-1990s, and are now proving highly successful.

A fibre laser generates a diffractionlimited beam by using doped monomode silica glass fibres as its laser active material. These fibres have a core diameter of $10-20 \mu \mathrm{m}$, and are doped with rare earth elements such as ytterbium or erbium. High-power output is realized through a combination of oscillators and amplifiers, and through the parallel coupling (fibre bundling) of those arrangements.
In contrast, the active medium of a disk laser is a YAG crystal disk that is $0.1 \mathrm{~mm}$ thick and around $15 \mathrm{~mm}$ in diameter. Because the disk is very thin it can be cooled efficiently, and is therefore able to deliver excellent beam quality. Ytterbium is used as the doping element because it can be strongly concentrated without causing quenching, which negatively affects the amplification and occurs for doping elements such as neodymium. Today, disk lasers with $4 \mathrm{~kW}$ of power from each disk are commercially available. The output power of disk lasers can be increased by putting additional disks into the resonator, and this does not change the beam quality of the system.

Although flashlamp-pumped pulsed Nd:YAG lasers are still widely used for precise spot- and seam-welding, diode-pumped fibre lasers are now entering this domain. For CW applications, diode-pumped fibre and disk lasers have completely exceeded the capabilities of flashlamp-pumped rod lasers, and their performance is improving all the time. Most applications today need a laser power of less than $10 \mathrm{~kW}$, but these new laser designs are ready for applications that require power levels of up to $100 \mathrm{~kW}$. For these high outputs, however, the optical components for beam transmission and focusing will need to be improved, and this will happen over time. Fibre and disk lasers have recently been finding important applications in the domain of $\mathrm{CO}_{2}$ laser cutting.

In the next few years, the diode laser will probably oust the fibre and disk laser as the most popular CW laser, at least at output ranges of up to several kilowatts. Investment costs and energy consumption will be the deciding factors here. However, if a high beam quality and laser power is required, fibre and disk lasers will remain the tools of choice.

Paul Seiler and Klaus Wallmeroth are at TRUMPF Laser, Aichhalderstr. 39, 78713 Schramberg, Germany; Kurt Mann is at TRUMPF Laser and Systemtechnik, Johann-Maus-Strasse 2, 71254 Ditzingen, Germany. e-mail:kurt.mann@de.trumpf.com 